Gefässchirurgie 2016 $21: 317-319$ DOI 10.1007/s00772-016-0179-x

Online publiziert: 10. August 2016

๑) Springer-Verlag Berlin Heidelberg 2016

CrossMark

F. Schönhoff · T. Carrel

Universitätsklinik für Herz- und Gefässchirurgie, Inselspital und Universität Bern, Bern, Schweiz

\title{
I-SWOT - ein wichtiger Beitrag zur Entscheidungsfindung in der Gefässmedizin
}

\author{
Kommentar zum Beitrag \\ Sachweh A, von Kodolitsch Y et al. (2016) \\ I-SWOT als Instrument zur individuell \\ optimierten Therapie bei thorakoabdomi- \\ nalem Aortenaneurysma. Effektiv, konform \\ und bedürfnisorientiert. Gefässchirurgie \\ doi:10.1007/s00772-016-0178-y
}

SWOT bezeichnet eine systematische Analyse von Stärken, Schwächen, Möglichkeiten und Risiken möglicher Strategien. Diese Art der Strategieplanung wird seit Jahrzehnten im Bereich der Geschäftsentwicklung benutzt und wurde bisher kaum im medizinischen Bereich verwendet.

Armin Sachweh, Yskert von Kodolitsch und Kollegen aus dem Universitätsklinikum Hamburg zeigen in ihrem Artikel eindrucksvoll die Relevanz dieser Methode für die Entscheidungsfindung im Hinblick auf die individuelle Behandlung von Patienten mit einer schwerwiegenden Aortenerkrankung.

Die Kollegen aus Hamburg sind im deutschsprachigen Raum eine der Vorreitergruppen im Bereich des „HeartTeam“-Konzepts und dessen praktischer Umsetzung. Sie zeigen mit diesem Beispiel die Stärken eines solchen Ansatzes: Eine schwierige Entscheidung wird durch diesen formalisierten Prozess auf verschiedene Teilaspekte heruntergebrochen und im Lichte der persönlichen Situation des Patienten aus verschiedenen Blickwinkeln reflektiert.

In einem kürzlich im New England Journal of Medicine publizierten Editorial weist Terri R. Fried darauf hin, dass Ärzte viel eher konkrete Ratschläge für ein bestimmtes Vorgehen geben, wenn die Evidenz für dieses Vorgehen groß ist [1]. Obwohl dies im ersten Moment nachvollziehbar erscheint, so muss man einsehen, dass der Patient gerade dann am meisten den Rat des Arztes benötigt, wenn die Evidenz schwach ist.

Eine besondere Stärke der hier vorgestellten Vorgehensweise ist sicher die der Nachvollziehbarkeit der Entscheidungsfindung, etwas, was gerade im klinischen Alltag zu wenig Gewicht erhält. Große Studien konnten zeigen, dass die Adhärenz an Leitlinien zu einem besseren klinischen Ergebnis bei den meisten behandelten Patienten führt. Eine Entscheidung entgegen den Empfehlungen der Leitlinien der großen Fachgesellschaften, insbesondere denen der Klasse I mit dem Evidenzgrad A, sollte daher nicht leichtfertig getroffen werden. Nichtsdestotrotz muss uns bewusst sein, dass die Medizin ihrem Naturell nach keine exakte Wissenschaft ist und Leitlinien - auch Klasse-I-Empfehlungen - sicher nie für alle Patienten die beste Behandlungsstrategie bedeuten. Eine genaue Kenntnis der Datenbasis der entsprechenden Leitlinie ist daher essenziell und muss integraler Bestandteil der Diskussion sein.

In unserem klinischen Umfeld zwingen wir uns selbst bei jeder Beratung, eine konkrete Behandlungsempfehlung für den Patienten abzugeben - umso mehr, wenn wir unsicher sind. Denn wenn wir als spezialisiertes, tertiäres Zentrum uns nicht zu einer Entscheidung durchringen können, wie soll man diese Entscheidung dann dem behandelnden Hausarzt, niedergelassenen Kollegen oder letztendlich dem Patienten zumuten? Des Weiteren muss man sich unseres Erachtens von der Idee befreien, dass es nur eine „rich- 
tige" Entscheidung gibt. Wir versuchen den Patienten zu vermitteln, dass es die „einzig richtige“ therapeutische Strategie bei pathologischen Befunden nicht gibt, sondern meist mehrere mögliche therapeutische Optionen zur Wahl stehen und sich der Patient deshalb zwischen diesen Optionen auch nicht „falsch“ entscheiden kann.

Ein wichtiger Aspekt der Arbeit von Sachweh et al., der zwar nicht explizit erwähnt wird, jedoch essenziell ist, ist der persönliche Kontakt mit dem Patienten. Nur im persönlichen Gespräch und bei Betreuung des Patienten über einen gewissen Zeitraum ist es möglich, die bei der SWOT-Analyse geforderten Einzelaspekte zu erfassen.

Die vorliegende Arbeit der Kollegen aus Hamburg stellt einen wichtigen Beitrag zur aktuellen Diskussion um den sog. „mündigen Patienten“ dar. Obwohl der Ansatz initial sehr technisch erscheint, wirft er uns doch auf das Essenzielle des ärztlichen Berufes zurück - einem Patienten mit aktivem Rat in einer schwierigen Situation zur Seite zu stehen und die gemeinsame Entscheidung in letzter Konsequenz mitzutragen.

\section{Korrespondenzadresse}

\section{PD Dr. F. Schönhoff}

Universitätsklinikfür Herz- und Gefässchirurgie, Inselspital und Universität Bern

Bern, Schweiz

florian.schoenhoff@insel.ch

Interessenkonflikt. F. Schönhoff und T. Carrel geben an, dass kein Interessenkonflikt besteht.

\section{Literatur}

1. Fried T (2016) Shared Decision Making-Finding the Sweet Spot. N Engl J Med 374:104-106

\section{W. Schäberle, Göppingen \\ Ultraschall in der Gefäßdiagnostik}

Therapieorientiertes Lehrbuch und Atlas

Berlin Heidelberg: Springer-Verlag

2016, 4. Auflage, 535 S., 575 Abb., (ISBN

978-3-662-47431-0), 149.99 EUR

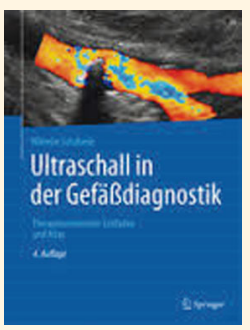

Ultraschall in der Gefäßdiagnostik erscheint als vierte Auflage im Springer Verlag und ist beinahe 20 Jahre nach der Ersterscheinung im Dezember 1997 immer noch ein inhaltlich vollständiges Ein-Autoren-Buch im Gefäßultraschall. Es spiegelt die langjährige Erfahrung und Expertise des Autors wider, der sich als Gefäßchirurg als Erster in der Deutschen Gesellschaft für Ultraschall in der Medizin (DEGUM) einen Namen gemacht hat. Es ist auf 535 Seiten ein in allen Einzelheiten ausführliches Lesebuch, das nach der Ultraschalldiagnostik die Brücke zur Therapie in der Gefäßmedizin schlägt. Die Ultraschalluntersuchung ist nicht Selbstzweck, sondern das moderne Stethoskop des Arztes, um eine Entscheidung für den weiteren Therapieverlauf direkt am und mit dem Patienten zu treffen. Viele Schemazeichnungen, Ultraschallabbildungen, aber auch ergänzende Bildaufnahmen aus CT und Angiographie erläutern die Befunde. Eigene Diagnosealgorithmen in der Shuntdiagnostik und bei der peripheren Verschlusskrankheit machen das Buch praxisnah. Methoden in der Verlaufskontrolle von Stents in der Carotis und in den peripheren Arterien wie die Verwendung der peak systolic velocity ratio (PSV ratio) wurden bisher so nicht publiziert. Mit 575 Abbildungen wird es zu Recht als therapieorientierter Leitfaden und Atlas bezeichnet. Das Buch ist angesichts der Hochglanzqualität und insbesondere der Vielzahl von beispielhaften Ultraschallbildern mit 149,99 Euro günstig.

Die vierte Auflage ist vorbildlich in Bildmaterial und Literaturverzeichnis aktualisiert, behält aber die übersichtliche, ursprüngliche Gliederung bei. Die Kontrastmittelsonographie (contrast enhanced ultrasound $=$ CEUS) ist nun als erweiterter Ultraschall im Gegensatz zur Vorauflage berücksichtigt. Bei der Nachkontrolle nach endovaskulären Aortenaneurysmaeingriffen hätte man sich zusätzlich einen Ausblick auf die Bildfusion von Ultraschall und beispielsweise CT gewünscht.

Zusammenfassend ein auch in der vierten Auflage herausragendes Buch, das in die Bibliothek jedes Gefäßmediziners gehört, der an einer zeiteffizienten, praktischen und vor allem therapieorientierten Ultraschalldiagnostik interessiert ist. Dem Buch ist wiederum eine weite Verbreitung zu wünschen.

K. Pfister (Regensburg) 
Hier steht eine Anzeige.

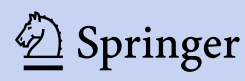

